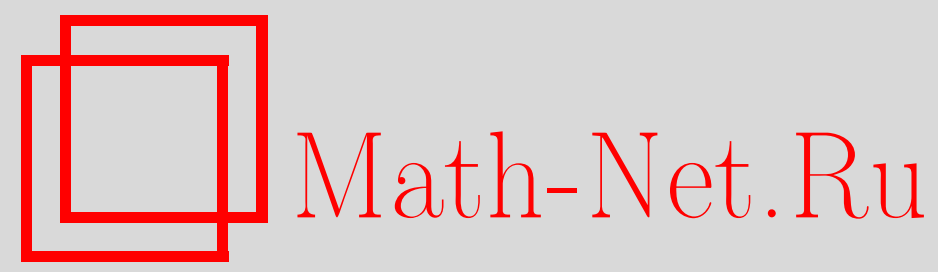

В. Н. Колокольцов, Обобщенные случайные блуждания в непрерывном времени (CTRW), субординация временами достижения и дробная динамика, Теория вероятн. и ее примен., 2008, том 53, выпуск 4, 684703

DOI: https://doi.org/10.4213/tvp2460

Использование Общероссийского математического портала MathNet.Ru подразумевает, что вы прочитали и согласны с пользовательским соглашением

http://www . mathnet.ru/rus/agreement

Параметры загрузки:

IP: 3.80 .181 .102

26 апреля 2023 г., 16:35:01

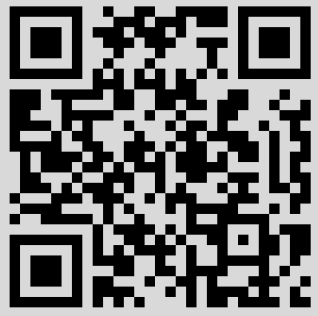




\section{ОБОБЩЕННЫЕ СЛУЧАЙНЫЕ БЛУЖДАНИЯ В НЕIРЕРЫВНОМ ВРЕМЕНИ (CTRW), СУБОРДИНАЦИЯ ВРЕМЕНАМИ ДОСТИЖЕНИЯ И ДРОБНАЯ ДИНАМИКА}

Получены функциональные предельные теоремы для случайных блужданий в непрерывном времени, в общем случае зависимых и зависящих от позиции скачков и времен ожидания, причем предельная аномальная диффузия определяется дробно-дифференциальными по времени эволюционными уравнениями. Вероятностная интерпретация обобщенной дробнодифференциальной динамики дана в терминах случайной замены времени (субординации) посредством процессов моментов достижения, соответствуюших возрастаюшим процессам Леви или Феллера.

Ключевые слова и фразы: дробно-дифференциальные уравнения, дробно-устойчивые распределения, аномальная диффузия, предельные теоремы, случайные блуждания в непрерывном времени, субординация, процессы моментов достижения.

1. Введение. Пусть $\left(X_{1}, T_{1}\right),\left(X_{2}, T_{2}\right), \ldots$ - последовательность независимых одинаково распределенных пар случайных величин, причем $X_{i} \in \mathbf{R}^{d}, T_{i} \in \mathbf{R}_{+}$(размеры скачков и времена ожидания между ними) и распределение каждой пары $\left(X_{i}, T_{i}\right)$ задается вероятностной мерой $\psi(d x d t)$ на $\mathbf{R}^{d} \times \mathbf{R}_{+}$. Положим

$$
N_{t}=\max \left\{n: \sum_{i=1}^{n} T_{i} \leqslant t\right\}
$$

Процесс

$$
S_{N_{t}}=X_{1}+X_{2}+\cdots+X_{N_{t}}
$$

называется случайным блужданием в непрерывном времени (общепринятая английская аббревиатура CTRW), порожденным $\psi$. Эти CTRW были введены в [19] и нашли многочисленные применения в физике и экономике (см., например, [25], [15], [3], [13], [17] и имеющиеся там ссылки).

* Department of Statistics, University of Warwick, Coventry CV4 7AL UK; Mocкoвский экономический институт (МЭИ); e-mail: v.kolokoltsov@warwick.ac.uk 
Особенно интересны те ситуации, когда $T_{i}$ принадлежат области притяжения $\beta$-устойчивого распределения, $\beta \in(0,1)$, а $X_{i}$ принадлежат области притяжения $\alpha$-устойчивого распределения, $\alpha \in(0,2)$. Предельные распределения подходящим образом нормированных сумм $S_{N_{t}}$ были описаны в [7] в случае независимых $T_{i}$ и $X_{i}$ (см. также [11]). В [5] была проанализирована скорость сходимости в более общей схеме с двойным массивом случайных величин, а в [15] соответствуюший функциональный предел был определен и было доказано, что его динамика задается дробно-дифференциальными уравнениями. Основные случаи зависимых $T_{i}$ и $X_{i}$ были далее проанализированы в [2] в рамках теории операторноустойчивых процессов (развитой в монографии [16]). В данной работе мы значительно расширяем теорию, допуская зависимость $\left(T_{n}, X_{n}\right)$ от текущего состояния процесса, т.е. рассматривая пространственно неоднородную ситуацию. Применяемый метод отличен от методов работ [7], [11], [16]. Он основан на конечно-разностной аппроксимации марковских полугрупп с непрерывным временем и опирается на ранее полученные в [8] результаты автора о пространственно неоднородных обобщениях устойчивьг процессов.

В [15] было замечено, что дробно-дифференциальная динамика возникает при субординации процессов Леви посредством моментов достижения устойчивых субординаторов Леви (т.е. посредством обрашенных траекторий устойчивых субординаторов). Неявно эта идея присутствовала уже в [21]. В качестве основы для наших предельных теорем мы развиваем в настоящей работе общую теорию субординации марковских процессов моментами достижения, показывая, как естественно эта процедура приводит к (обобщенным) дробно-дифференциальным уравнениям. В частности, обрашаясь критически к замечанию, сделанному в [15], что метод работы [21], восходяший к [19], «does not identify the limit process» (не позволяет определить предельный процесс), мы даем строгую вероятностную интерпретацию интуитивно ясных, но довольно формальных вычислений из [21].

Настоящая работа организована следующим образом. В следующем пункте мы демонстрируем наш подход к предельным теоремам для CTRW, доказав простые (но тем не менее, по-видимому, новые) предельные теоремы для зависящих от позиции (пространственно неоднородных) случайных блужданий с размерами скачков из областей притяжения устойчивых законов. В п. 3 эти результаты обобщаются на разномасштабные по двум направлениям случайные блуждания, необходимые для дальнейшего анализа CTRW. Пункт 4 (независимый от остального и имеющий независимый интерес) посвящен общей теории субординации моментами достижения. В п. 5, соединяя две части теории из пп. 3 и 4 , мы доказываем наши основные результаты о CTRW.

Зафиксируем теперь некоторые (довольно стандартные) обозначе- 
ния, используемые в работе. Для локально компактного пространства $X$ обозначаем через $C(X)$ банахово пространство ограниченных непрерывных функций на $X$ (снабженное равномерной нормой), а через $C_{\infty}(X)$ - его замкнутое подпространство, состоящее из функций, исчезающих на бесконечности. Через $(f, \mu)$ обозначается обычное спаривание $\int f(x) \mu(d x)$ между функциями и мерами. Под непрерывным семейством переходных вероятностей в $X$ понимается, как обычно, семейство $p(x ; d y)$ вероятностных мер на $X$, зависящее непрерывно от $x \in X$, причем это семейство считается снабженным слабой топологией: $\mu_{n} \rightarrow \mu$ при $n \rightarrow \infty$, если $\left(f, \mu_{n}\right) \rightarrow(f, \mu)$ при $n \rightarrow \infty$ для каждой $f \in C(X)$.

Для меры $\mu(d y)$ в $\mathbf{R}^{d}$ и положительного числа $h$ будем обозначать через $\mu(d y / h)$ промасштабированную меру, определенную ее действием

$$
\int g(z) \mu(d z / h)=\int g(h y) \mu(d y)
$$

на функции $g \in C\left(\mathbf{R}^{d}\right)$.

Заглавные латинские буквы $\mathbf{E}$ и $\mathbf{P}$ зарезервированы для обозначения математического ожидания и вероятности. Функция $\delta(x)$ - это обычная точечная мера Дирака.

2. Предельные теоремы для пространственно неоднородных случайных блужданий. Для вектора $y \in \mathbf{R}^{d}$ будем обозначать через $\bar{y}$ его нормализацию: $\bar{y}=y /|y|$, где $|y|$ - обычная евклидова норма вектора $y$.

Выберем произвольное $\alpha \in(0,2)$. Пусть $S: \mathbf{R}^{d} \times S^{d-1} \rightarrow \mathbf{R}_{+}-$ непрерьвная неотрицательная функция, симметричная по второму аргументу, т.е. $S(x, y)=S(x,-y)$. Такая функция задает семейство $\alpha$ устойчивых $d$-мерных симметричных случайных векторов (зависящих от $x \in \mathbf{R}^{d}$ ) посредством их характеристических функций $\phi_{x}$ таких, что

$$
\ln \phi_{x}(p)=\int_{0}^{\infty} \int_{S^{d-1}}\left(e^{i(p, \xi)}-1-\frac{i(p, \xi)}{1+\xi^{2}}\right) \frac{d|\xi|}{|\xi|^{1+\alpha}} S(x, \bar{\xi}) d_{S} \bar{\xi}
$$

где $d_{S}$ - лебегова мера на сфере $S^{d-1}$. Хорошо известно, что (2) можно переписать в виде

$$
\ln \phi_{x}(p)=C_{\alpha} \int_{S^{d-1}}|(p, \bar{\xi})|^{\alpha} S(x, \bar{\xi}) d_{S} \bar{\xi}
$$

с некоторой постоянной $C_{\alpha}$.

3 а м е ч а н и е 1. Нет видимых препятствий для обобщения излагаемой здесь теории на несимметричные устойчивые законы. Однако работа с симметричными законами существенно упрощает формулы.

Теорема 2.1. Предположим, что

$$
C_{1} \leqslant \int_{S^{d-1}}|(\bar{p}, s)|^{\alpha} S(x, s) d_{S} s \leqslant C_{2}
$$


для всех $p$ с некоторыми постоянными $C_{1}, C_{2}$, а также ито $S(x, s)$ имеет ограниченные производные по $x$ до порядка $q \geqslant 3$ включительно (если $\alpha<1$, то достаточно считать, что $q \geqslant 2$ ). Тогда псевдоди $\phi$ ференииальный оператор

$$
L f(x)=\ln \phi_{x}\left(\frac{1}{i} \frac{\partial}{\partial x}\right) f(x)=\int_{0}^{\infty} \int_{S^{d-1}}(f(x+y)-f(x)) \frac{d|y|}{|y|^{1+\alpha}} S(x, \bar{y}) d_{S} \bar{y}
$$

порождает Феллеровскую полугруппу $T_{t}$ в $C_{\infty}\left(\mathbf{R}^{d}\right)$, причем пространство $C^{q-1}\left(\mathbf{R}^{d}\right) \cap C_{\infty}\left(\mathbf{R}^{d}\right)$ является инвариантной существенной областью ее генератора.

Этот результат доказан в [8] и [9].

3 а м е ч а н и е 2. В [8] показано также, что полугруппа $T_{t}$ имеет гладкую плотность переходной вероятности (функцию Грина).

Обозначим $Z_{x}(t)$ феллеровский процесс, построенный по полугруппе $T_{t}$. Мы займемся сейчас дискретными приближениями к $T_{t}$ и $Z_{x}(t)$.

Начнем со следующего технического факта.

Предложение 2.1. Пусть $p(x ; d y)$ - непрерывное семейство переходных вероятностей в $\mathbf{R}^{d}$ из нормальной области притяжения устойчивого закона вида (2). Точнее, предположим, что для произвольного открытого множества $\Omega \subset S^{d-1}$ с границей нулевой меры Лебега

$$
\int_{|y|>n} \int_{\bar{y} \in \Omega} p(x ; d y) \sim \frac{1}{\alpha n^{\alpha}} \int_{\Omega} S(x, s) d_{S} s, \quad n \rightarrow \infty,
$$

т.е. отношение двух частей этой формуль стремится $\propto$ единиче при $n \rightarrow \infty$ равномерно по $x$. Предположим также, что $p(x ;\{0\})=0$ nрu всех $x$. Тогда

$$
\min \left(1,|y|^{2}\right) p\left(x ; \frac{d y}{h}\right) h^{-\alpha} \longrightarrow \min \left(1,|y|^{2}\right) \frac{d|y|}{|y|^{\alpha+1}} S(x, \bar{y}) d_{S} \bar{y}, \quad h \rightarrow 0,
$$

где обе части являются конечными мерами в $\mathbf{R}^{d} \backslash\{0\}$ и сходимость слабая, но равномерная по $x \in \mathbf{R}^{d}$. Если $\alpha<1$, то и соотношение

$$
\min (1,|y|) p\left(x ; \frac{d y}{h}\right) h^{-\alpha} \longrightarrow \min (1,|y|) \frac{d|y|}{|y|^{\alpha+1}} \int_{\Omega} S(x, \bar{y}) d_{S} \bar{y}, \quad h \rightarrow 0,
$$

в том же смысле выполнено.

3 а м е ч а н и е 3 . Так как предельная мера абсолютно непрерывна по отношению к лебеговой, то равномерная слабая сходимость означает просто, что меры всякого открытого множества с границей нулевой лебеговой меры сходятся равномерно по $x$. 
Д ок а з а т е ль с т в о. Согласно (4),

$$
\int_{|z|>A} \int_{\bar{z} \in \Omega} p\left(x ; \frac{d z}{h}\right) h^{-\alpha}=\int_{|y|>A / h} \int_{\bar{y} \in \Omega} p(x ; d y) h^{-\alpha} \sim \frac{1}{\alpha A^{\alpha}} \int_{\Omega} S(x, s) d_{S} s
$$

при $h \rightarrow 0$. Значит,

$$
\int_{A<|z|<B} \int_{\bar{z} \in \Omega} p\left(x ; \frac{d z}{h}\right) h^{-\alpha} \longrightarrow \int_{A}^{B} \frac{d|z|}{|z|^{\alpha+1}} \int_{\Omega} S(x, s) d_{S} s .
$$

Поэтому $p(x ; d z / h) h^{-\alpha}$ слабо сходится к $|z|^{-(\alpha+1)} d|z| S(x, z /|z|) d_{S}(z /|z|)$ на всяком множестве, отделенном от нуля. Легко видеть, что (5) вытекает теперь из равномерной оценки

$$
\int_{|y|<\varepsilon} \min \left(1,|y|^{2}\right) p\left(x ; \frac{d y}{h}\right) h^{-\alpha} \leqslant C \varepsilon^{2-\alpha}
$$

с некоторой постоянной $C$. Чтобы доказать (6), заметим, что выполнено неравенство $\int_{|y|>n} p(x ; d y) \leqslant C n^{-\alpha}$, где постоянная $C$ одна и та же для всех $x$ и $n>0$ (действительно, это выполнено для больших $n$ согласно (4) и распространяется на все $n$, поскольку $p(x ; d y)$ - это вероятностные меры и, значит, ограничены). Следовательно, для произвольного $\varepsilon<1$ выполнено равенство

$$
\int_{|y|<\varepsilon} \min \left(1,|y|^{2}\right) p\left(x ; \frac{d y}{h}\right) h^{-\alpha}=\int_{|z|<\varepsilon / h} h^{2}|z|^{2} p\left(x ; \frac{d y}{h}\right) h^{-\alpha} .
$$

Представляя последний интеграл в виде счетной суммы интегралов по областям $\varepsilon /\left(2^{k+1} h\right)<y \leqslant \varepsilon /\left(2^{k} h\right)$, его можно оценить выражением

$$
\sum_{k=0}^{\infty} h^{2}\left(\frac{\varepsilon}{2^{k} h}\right)^{2} h^{-\alpha} C h^{\alpha} \cdot 2^{\alpha(k+1)} \varepsilon^{-\alpha}=\sum_{k=0}^{\infty} C \varepsilon^{2-\alpha} \cdot 2^{\alpha} \cdot 2^{-(2-\alpha) k} .
$$

Это и влечет (6), ибо сумма в правой части сходится.

Указанное уточнение в случае $\alpha<1$ получается аналогично.

Предложение 2.1 доказано.

Рассмотрим скачкообразный марковский процесс $Z^{h}(t)$, порожденный генератором

$$
\left(L_{h} f\right)(x)=\frac{1}{h^{\alpha}} \int(f(x+h y)-f(x)) p(x ; d y) .
$$

При каждом $h$ оператор $L_{h}$ ограничен в $C_{\infty}\left(\mathbf{R}^{d}\right)$ и, следовательно, однозначно определяет феллеровскую полугруппу. Вероятностная интерпретация процесса $Z^{h}(t)$ такова. Начавшись в точке $x$, процесс ждет случайное $h^{-\alpha}$-экспоненциальное время $\tau$ (т.е. $\mathbf{P}\{\tau>t\}=\exp \left(-t h^{-\alpha}\right)$ ) 
и затем переходит в $x+h Y$, где $Y$ распределено согласно $p(x ; d y)$. Далее то же повторяется из точки $x+h Y$ и т.д. В том случае, когда $p$ не зависит от $x$, процесс

$$
Z^{h}(t)=h\left(Y_{1}+\cdots+Y_{N_{t}}\right)
$$

является нормированным случайным блужданием, число скачков $N_{t}$ которого - это пуассоновский процесс с параметром $h^{-\alpha}$, так что $\mathbf{E} N_{t}=t h^{-\alpha}$. В частности, $n=N_{t} \sim t h^{-\alpha}$ при мальх $h$, так что $Z^{h}(1) \sim n^{-1 / \alpha}\left(Y_{1}+\cdots+Y_{n}\right)$.

Теорема 2.2. Полугруппа $T_{t}^{h}$, порожденная $L_{h}$, сходится $\kappa$ полугруппе $T_{t}$, порожденной $L . B$ частности, соответствуюшие прочессь сходятся в смысле конечномерных распределений.

3 а м е ч н и е 4. Всюду в этой работе мы работаем исключительно со сходимостью полугрупп. Однако, согласно известным результатам (см., например, [6, теорема 19.25]), для феллеровских процессов такая сходимость эквивалентна сходимости распределений на траекториях в подходяшем пространстве Скорохода.

Д ок а з т ел ь с т в. Согласно (7),

$$
\left(L_{h} f\right)(x)=\frac{1}{h^{\alpha}} \int(f(x+z)-f(x)) p\left(x ; \frac{d z}{h}\right)
$$

что ввиду предложения 2.1 сходится к $L f(x)$ при $h \rightarrow 0$ равномерно по $x$ для $f \in C_{\infty}\left(\mathbf{R}^{d}\right) \cap C^{2}\left(\mathbf{R}^{d}\right)$. Согласно известному результату (см., например, [14]), сходимость генераторов на сушественной области определения предельного генератора влечет за собой сходимость полугрупп. Теорема 2.2 доказана.

Следующий результат касается приближений с неслучайным числом скачков. Зададим процесс $S_{x}^{h}(t)=S_{x}^{h}([t])$ (квадратная скобка обозначает целую часть числа) формулой

$$
S_{x}^{h}(0)=x, \quad S_{x}^{h}(1)=x+h Y_{1}, \quad \ldots, \quad S_{x}^{h}(j)=S_{x}^{h}(j-1)+h Y_{j}, \quad \ldots,
$$

где каждое $Y_{j}$ распределено согласно $p\left(S_{j-1} ; d y\right)$. Если $p(x ; d y)$ не зависит от $x$, то

$$
S_{x}^{h}(n)=x+h\left(Y_{1}+\cdots+Y_{n}\right)
$$

- обычное случайное блуждание.

Мы сравним феллеровский процесс $Z_{x}(t)$ на произвольном фиксированном временном интервале $\left[0, t_{0}\right]$ с дискретными приближениями $S_{x}^{h}(t / \tau)$, где число скачков $n=t / \tau$ связано с масштабным параметром $h$ соотношением $\tau=h^{\alpha}$.

Теорема 2.3. При условиях теоремь 2.1 и предложения 2.1 E $f\left(S_{x}^{h}(t / \tau)\right)$ cходится $\kappa T_{t} f(x)$ nрu $\tau=h^{\alpha} \rightarrow 0$ для каждой $f \in C_{\infty}\left(\mathbf{R}^{d}\right)$ 
равномерно по $t \in\left[0, t_{0}\right]$. В частности, прочессы $S_{x}^{h}(t / \tau)$ сходятся $\mathcal{K}$ $Z_{x}(t)$ в смысле конечномерных распределений.

Д о к а з а т е л в с т в о. Согласно теореме 2.1, достаточно доказать требуемую сходимость только для $f \in C^{2}\left(\mathbf{R}^{d}\right) \cap C_{\infty}\left(\mathbf{R}^{d}\right)$. Выберем такую функцию $f$. Обозначим $f_{k}(x)=\mathbf{E} f\left(S_{x}^{h}(k)\right)$. Тогда, в силу марковского свойства, $f_{k}=R_{h}^{k} f$, где оператор $R_{h}$ определяется формулой

$$
R_{h} f(x)=\int f(x+h y) p(x ; d y) .
$$

Ясно, что каждый $R_{h}$ - сохраняющий положительность оператор сжатия на $C_{\infty}\left(\mathbf{R}^{d}\right)$. С другой стороны, рекуррентное уравнение $f_{k}=R f_{k-1}$ может быть записано в виде

$$
\frac{f_{k}(x)-f_{k-1}(x)}{\tau}=h^{-\alpha} \int\left(f_{k-1}(x+h y)-f_{k-1}(x)\right) p(x ; d y) .
$$

А это есть приближение в дискретном времени к уравнению

$$
\frac{\partial f}{\partial t}=L f
$$

на функциях $f$, принадлежащих $C^{2}\left(\mathbf{R}^{d}\right) \cap C_{\infty}\left(\mathbf{R}^{d}\right)$ (и дифференцируемых по $t$ ). Так как эта разностная схема устойчива (ибо решается оператором сжатия $R_{h}^{n}$ ), а решение уравнения (9) однозначно определено и сохраняет пространство $C^{2}\left(\mathbf{R}^{d}\right) \cap C_{\infty}\left(\mathbf{R}^{d}\right)$ (по теореме 2.1 ), то из стандартных (и легко доказываемых) обших фактов теории разностных схем (см. [22]) вытекает, что решения конечномерных аппроксимаций сходятся к решению уравнения (9). Теорема 2.3 доказана.

В том случае, когда $p$ не зависит от $x$, теорема 2.3 преврашается в известный факт о сходимости случайных блужданий с распределением скачков из области нормального притяжения устойчивого закона к соответствующему устойчивому процессу Леви.

3. Случайные блуждания с разным масштабом по разным направлениям. Для применения развитой теории к CTRW необходимо ее обобщение на случай разномасштабных блужданий, которое мы сейчас и обсудим.

Рассмотрим процесс в $\mathbf{R}^{d} \times \mathbf{R}_{+}$, заданный генератором

$$
\begin{aligned}
\mathscr{L} f(x, u)= & \int_{0}^{\infty} \int_{S^{d-1}}(f(x+y, u)-f(x, u)) \frac{d|y|}{|y|^{1+\alpha}} S(x, u, \bar{y}) d_{S} \bar{y} \\
& +\int_{0}^{\infty}(f(x, u+v)-f(x, u)) \frac{1}{v^{1+\beta}} w(x, u) d v .
\end{aligned}
$$

Следующий результат (как и его доказательство) является непосредственным обобщением теоремы 2.1 . 
Теорема 3.1. Пусть

$$
C_{1} \leqslant \int_{S^{d-1}}|(\bar{p}, s)|^{\alpha} S(x, u, s) d_{S} s \leqslant C_{2}, \quad C_{1} \leqslant w(x, u) \leqslant C_{2}
$$

с некоторыми постоянными $C_{1}, C_{2}, a S(x, u, s) u w(x, u)$ имеют ограниченные производные по $x$ и и до порядка $q \geqslant 3$ включительно. Тогда псевдодифференииальный оператор (10) порождает феллеровскую полугруппу $\mathscr{T}_{t}$ в $C_{\infty}\left(\mathbf{R}^{d} \times \mathbf{R}_{+}\right)$(непрерывные функиии вплоть до гранииь), причем пространство $\left(C^{q-1} \cap C_{\infty}\right)\left(\mathbf{R}^{d} \times \mathbf{R}_{+}\right)$является инвариантной существенной областью генератора, а значит, и феллеровский прочесс $(Y, V)(t){ }_{\text {в }} \mathbf{R}^{d} \times \mathbf{R}_{+}$.

Получим теперь соответствующее обобщение теорем $2.2,2.3$.

Теорема 3.2. Пусть $p(x, u ; d y d v)$ - непрерывное семейство переходных вероятностей в $\mathbf{R}^{d} \times \mathbf{R}_{+}$, симметричное по отношению $\kappa$ отражению $y \mapsto-y$ и такое, ито $p\left(x, u ;\{0\} \times \mathbf{R}_{+}\right)+p\left(x, u ; \mathbf{R}^{d} \times\{0\}\right)=0$. Допустим также, что проекиии принадлежат областям нормального притяжения устойчивых законов, а точнее, что равномерно по $(x, u)$

$$
\begin{aligned}
& \int_{|y|>n} \int_{\bar{y} \in \Omega} p(x, u ; d y d v) \sim \frac{1}{\alpha n^{\alpha}} \int_{\Omega} S(x, u, s) d_{S} s, \quad n \rightarrow \infty \\
& \int_{v>n} \int_{|y|>A} p(x, u ; d y d v) \sim \frac{1}{\beta n^{\beta}} w(x, u, A), \quad n \rightarrow \infty
\end{aligned}
$$

при каждом $A \geqslant 0$, где $w$ - измеримая функиия трех аргументов такая, чmo

$$
w(x, u, 0)=w(x, u), \quad \lim _{A \rightarrow \infty} w(x, u, A)=0
$$

(таким образом, $w(x, u, A)$ является мерой в $\mathbf{R}_{+}$при всех $\left.x, u\right)$.

Рассмотрим скачкообразные прочессы, порожденные

$$
\left(\mathscr{L}_{\tau} f\right)(x, u)=\frac{1}{\tau} \int\left(f\left(x+\tau^{1 / \alpha} y, u+\tau^{1 / \beta} v\right)-f(x, u)\right) p(x, u ; d y d v)
$$

Тогда Феллеровские полугруппы $\mathscr{T}_{t}^{h}$ в $C_{\infty}\left(\mathbf{R}^{d} \times \mathbf{R}_{+}\right)$этих прочессов (которые являются феллеровскими прочессами, поскольку $\mathscr{L}_{h}$ ограничены в $C_{\infty}\left(\mathbf{R}^{d} \times \mathbf{R}_{+}\right)$при каждом $\left.h\right)$ сходятся $к$ полугруппе $\mathscr{T}_{t}$.

Д о к а з а т е л в с т в о. Как и в предложении 2.1, из (11), (12) следует, что равномерно по $x, u$ при $h \rightarrow 0$

$$
\begin{aligned}
& \min \left(1,|y|^{2}\right) \int_{0}^{\infty} p\left(x, u ; \frac{d y d v}{h}\right) h^{-\alpha} \longrightarrow \min \left(1,|y|^{2}\right) \frac{d|y|}{|y|^{\alpha+1}} S(x, \bar{y}) d_{S} \bar{y} \\
& \min (1, v) \int_{|y|>A} p\left(x, u ; \frac{d y d v}{h}\right) h^{-\beta} \longrightarrow \min (1, v) w(x, u, A) \frac{d v}{v^{\beta+1}}
\end{aligned}
$$


Далее, взяв $f \in\left(C^{2} \cap C_{\infty}\right)\left(\mathbf{R}^{d} \times \mathbf{R}_{+}\right)$и записав

$$
\mathscr{L}_{\tau} f(x, u)=I+I I
$$

где

$$
\begin{aligned}
I=\frac{1}{\tau} & \int\left(f\left(x+\tau^{1 / \alpha} y, u\right)-f(x, u)\right) p(x, u ; d y d v) \\
+ & \frac{1}{\tau} \int\left(f\left(x, u+\tau^{1 / \beta} v\right)-f(x, u)\right) p(x, u ; d y d v), \\
I I=\frac{1}{\tau} \int & {\left[\left(f\left(x+\tau^{1 / \alpha} y, u+\tau^{1 / \beta} v\right)\right.\right.} \\
& \left.\left.\quad-f\left(x+\tau^{1 / \alpha} y, u\right)\right)-\left(f\left(x, u+\tau^{1 / \beta} v\right)-f(x, u)\right)\right] p(x, u ; d y d v),
\end{aligned}
$$

замечаем, что, как и при доказательстве теоремы 2.2 , из соотношений (15) и (16) (последнее с $A=0$ ) вытекает сходимость $I$ к $\mathscr{L} f(x, u)$, равномерная по $x, u$. Поэтому для завершения доказательства надо лишь доказать сходимость $I I$ к нулю при $\tau \rightarrow 0$. Имеем

$$
I I=\int\left(g\left(x+\tau^{1 / \alpha} y, u, v\right)-g(x, u, v)\right) p\left(x, u ; \frac{d y d v}{\tau^{1 / \beta}}\right) \frac{1}{\tau},
$$

где $g(x, u, v)=f(x, u+v)-f(x, u)$.

Согласно свойствам $f$,

$$
\begin{aligned}
|g(x, u, v)| & \leqslant C \min (1, v)\left(\max \left|\frac{\partial f}{\partial u}\right|+\max |f|\right) \leqslant \widetilde{C} \min (1, v), \\
\left|\frac{\partial g}{\partial x}(x, u, v)\right| & \leqslant C \min (1, v)\left(\max \left|\frac{\partial^{2} f}{\partial u \partial x}\right|+\max \left|\frac{\partial f}{\partial x}\right|\right) \leqslant \widetilde{C} \min (1, v)
\end{aligned}
$$

с некоторыми постоянными $C$ и $\widetilde{C}$. Значит, в силу (16) и (13) для произвольного $\varepsilon>0$ существует такое $A$, что

$$
\int_{|y|>A}\left(g\left(x+\tau^{1 / \alpha} y, u, v\right)-g(x, u, v)\right) p\left(x, u ; \frac{d y d v}{\tau^{1 / \beta}}\right) \frac{1}{\tau}<\varepsilon ;
$$

а с другой стороны, для произвольного $A$

$$
\int_{|y|<A}\left(g\left(x+\tau^{1 / \alpha} y, u, v\right)-g(x, u, v)\right) p\left(x, u ; \frac{d y d v}{\tau^{1 / \beta}}\right) \frac{1}{\tau} \leqslant \tau^{1 / \alpha} A \kappa
$$

с некоторой постоянной $\kappa$, так что $I I$ можно сделать сколь угодно малым выбором вначале большого $A$, а затем достаточно малого $\tau$.

Определим теперь процесс $(Y, V)_{x, u}^{\tau}(t / \tau)=(Y, V)_{x, u}^{\tau}([t / \tau])$, где

$$
\begin{aligned}
& (Y, V)_{x, u}^{\tau}(0)=(x, u), \quad(Y, V)_{x, u}^{\tau}(1)=\left(x+\tau^{1 / \alpha} Y_{1}, u+\tau^{1 / \beta} V_{1}\right), \ldots \\
& (Y, V)_{x, u}^{\tau}(j)=(Y, V)_{x, u}^{\tau}(j-1)+\left(\tau^{1 / \alpha} Y_{j}, \tau^{1 / \beta} V_{j}\right), \ldots,
\end{aligned}
$$


а каждая пара $\left(Y_{j}, V_{j}\right)$ распределена согласно $p\left((Y, V)_{x, u}^{\tau}(j-1) ; d y d v\right)$. Если $p(x, u ; d y d v)$ не зависит от $x, u$, то

$$
(Y, V)_{x, u}^{\tau}(n)=(x, u)+\left(\tau^{1 / \alpha}\left(Y_{1}+\cdots+Y_{n}\right), \tau^{1 / \beta}\left(V_{1}+\cdots+V_{n}\right)\right)
$$

Ввиду теоремы 3.2 , следующий результат получается буквально так же, как и теорема 2.3 .

Теорема 3.3. При условиях теорем 3.1 и 3.2 линейные сжатия E $f\left((Y, V)_{x, u}^{\tau}(t / \tau)\right)$ в $C_{\infty}\left(\mathbf{R}^{d} \times \mathbf{R}_{+}\right)$сходятся $\kappa$ полугруппе $\mathscr{T}_{t} f(x, u)$ nрочесса $(Y, V)(t)$ равномерно по $t \in\left[0, t_{0}\right]$ nрu $\tau \rightarrow 0$.

4. Субординация процессами моментов достижения и дробно-дифференциальная динамика. Пусть $X(u), u \geqslant 0$, - субординатор Леви, т.е. непрерывный справа возрастающий процесс с независимыми одинаково распределенными прирашениями (согласованный с некоторой полной непрерывной справа фильтрацией вероятностного пространства) с генератором

$$
A f(x)=\int_{0}^{\infty}(f(x+y)-f(x)) \nu(d y)+a \frac{\partial f}{\partial x}
$$

где $a \geqslant 0$, а $\nu$ - такая борелевская мера на $\{y>0\}$, что

$$
\int_{0}^{\infty} \min (1, y) \nu(d y)<\infty
$$

Рассмотрим процесс, полученный обращением (в смысле обратной функции) траекторий этого процесса, или, иначе говоря, процесс моментов достижения $Z(t)$, определенный как

$$
Z_{X}(t)=Z(t)=\inf \{u: X(u)>t\}=\sup \{u: X(u) \leqslant t\}
$$

и, разумеется, также являющийся возрастающим непрерывным справа процессом. Чтобы сделать дальнейший анализ более прозрачным (и избежать дополнительных усложнений самого общего случая), будем далее предполагать, что существуют такие $\varepsilon>0$ и $\beta \in(0,1)$, что

$$
\nu(d y) \geqslant y^{1+\beta} d y, \quad 0<y<\varepsilon .
$$

Для удобства ссылок в следующем утверждении мы приводим (без доказательства) элементарные известные свойства процесса $X(u)$.

Предложение 4.1. Пусть выполнено условие (19). Тогда:

(i) прочесс $X(u)$ почти наверное возрастает в каждой точке, m.e. не является постоянным ни на каком конечном интервале времени; 
(ii) распределение прочесса $X(u)$ при $u>0$ имеет плотность, скажем, $G(u, y)$, обрашаюшуюся в нуль при $y<0$, бесконечно дифференцируемую по обеим переменным и удовлетворяюшую уравнению

$$
\frac{\partial G}{\partial u}=A^{\star} G
$$

где $A^{\star}$, двойственньй оператор $\propto$, имеет вид

$$
A^{\star} f(x)=\int_{0}^{\infty}(f(x-y)-f(x)) \nu(d y)-a \frac{\partial f}{\partial x}
$$

(iii) продолженная нулем на полупространство $\{u<0\}$, локально интегрируемая функиия $G(u, y)$ на $\mathbf{R}^{2}$ определяет обобщенную функиию,совпадаюшую с бесконечно гладкой функиией вне точки $(0,0)$ u удовлетворяющую в смысле обобщенных функций (распределений) уравнению

$$
\frac{\partial G}{\partial u}=A^{\star} G+\delta(u) \delta(y)
$$

Следствие 1. Пусть выполнено условие (19). Тогда:

(i) прочесс $Z(t)$ почти наверно непрерывен и $Z(0)=0$;

(ii) распределение $Z(t)$ имеет непрерывно дифференчируемую плотность вероятности $Q(t, u)$ при $u>0$ :

$$
Q(t, u)=-\frac{\partial}{\partial u} \int_{-\infty}^{t} G(u, y) d y
$$

Д ок а з а т л ь с т о. Утверждение (i) вытекает из предложения 4.1 (i). Для доказательства (ii) замечаем, что

$$
\mathbf{P}\{Z(t) \leqslant u\}=\mathbf{P}\{X(u) \geqslant t\}=\int_{t}^{\infty} G(u, y) d y=1-\int_{0}^{t} G(u, y) d y
$$

откуда в силу дифференцируемости $G$ вытекает (22). Подчеркнем для ясности, что $(22)$ определяет $Q(t, u)$ как гладкую функцию при всех $t$, коль скоро $u>0$, и что $Q(t, u)=0$ при $t \leqslant 0$ и $u>0$.

Теорема 4.1. При условии (19) плотность $Q$ удовлетворяет уравнению

$$
A^{\star} Q=\frac{\partial Q}{\partial u}
$$

при $u>0$, где $A^{\star}$ действует на переменную $t, u$ граничному условию

$$
\lim _{u \rightarrow 0_{+}} Q(t, u)=-A^{\star} \theta(t)
$$

где $\theta(t)$ - индикаторная функиия, равная единиче (соответственно нулю) для положительных (соответственно отричательных) значений $t$. Продолженная нулем в полупространство $\{u<0\}$, Функиия $Q$ 
удовлетворяет в смысле обобщенных функиий уравнению

$$
A^{\star} Q=\frac{\partial Q}{\partial u}+\delta(u) A^{\star} \theta(t) .
$$

Кроме того, (поточечная) производная $\frac{\partial Q}{\partial t}$ также удовлетворяет уравнению (23) при и 0 и удовлетворяет уравнению

$$
A^{\star} \frac{\partial Q}{\partial t}=\frac{\partial}{\partial u} \frac{\partial Q}{\partial t}+\delta(u) \frac{d}{d t} A^{\star} \theta(t)
$$

в смысле распределений.

3 а м е ч а н и е 5 . В случае $\beta$-устойчивого субординатора $X(u)$ с генератором

$$
A f(x)=-\frac{1}{\Gamma(-\beta)} \int_{0}^{\infty}(f(x+y)-f(x)) y^{-1-\beta} d y
$$

имеем

$$
A=-\frac{d^{\beta}}{d(-x)^{\beta}}, \quad A^{\star}=-\frac{d^{\beta}}{d x^{\beta}}
$$

(эти уравнения можно рассматривать как определения дробных производных; с мотивацией и эквивалентными представлениями можно ознакомиться по книгам [18] и [20]; краткий, но содержательный обзор приведен также в приложении к статье [21]), и в этом случае уравнение (25) принимает вид

$$
\frac{d^{\beta} Q}{d t^{\beta}}+\frac{\partial Q}{\partial u}=\delta(u) \frac{t^{-\beta}}{\Gamma(1-\beta)}
$$

что совпадает с уравнением (В14) из [21]. Это замечание наводит на мысль называть операторы типа (17) обобщенными дробнодифференциальными.

Д о к а з а т е л в с т в о. Заметим, что в силу $(22),(20)$ и благодаря коммутативности операторов интегрирования и $A^{\star}$ выполнены равенства

$$
\begin{aligned}
Q(t, u) & =-\int_{-\infty}^{t} \frac{\partial}{\partial u} G(u, y) d y=-\int_{-\infty}^{t}\left(A^{\star} G(u, \cdot)\right)(y) d y \\
& =-A^{\star} \int_{-\infty}^{t} G(u, y) d y
\end{aligned}
$$

Это влечет за собой (23) (достаточно продифференцировать по $u$ и снова использовать (22)) и $(24)$, ибо $G(0, y)=\delta(y)$.

Предположим теперь, что $Q$ продолжена нулем в полупространство $\{u<0\}$. Пусть $\phi-$ пробная функция в $\mathbf{R}^{2}$, бесконечно дифференцируемая и имеюшая компактный носитель. Тогда в смысле обобщенных 
функций

$$
\begin{aligned}
& \left(\left(\frac{\partial}{\partial u}-A^{\star}\right) Q, \phi\right)=\left(Q,\left(-\frac{\partial}{\partial u}-A\right) \phi\right) \\
& \quad=\lim _{\varepsilon \rightarrow 0} \int_{\varepsilon}^{\infty} d u \int_{\mathbf{R}} d t Q(t, u)\left(-\frac{\partial}{\partial u}-A\right) \phi(t, u) \\
& \quad=\lim _{\varepsilon \rightarrow 0}\left[\int_{\varepsilon}^{\infty} d u \int_{\mathbf{R}} d t \phi(t, u)\left(\frac{\partial}{\partial u}-A^{\star}\right) Q(t, u)+\int_{\mathbf{R}} \phi(t, \varepsilon) Q(t, \varepsilon) d t\right]
\end{aligned}
$$

Первый член здесь обращается в нуль в силу (23). Следовательно, в силу (24),

$$
\left(\left(\frac{\partial}{\partial u}-A^{\star}\right) Q, \phi\right)=-\int_{\mathbf{R}} \phi(t, 0) A^{\star} \theta(t) d t
$$

что влечет за собой (25). Требуемые свойства производной $\frac{\partial Q}{\partial t}$ вытекают подобным же образом из представления

$$
\frac{\partial Q}{\partial t}(t, u)=-\frac{\partial G}{\partial u}(u, t)
$$

Например, при $u>0$

$$
A^{\star} \frac{\partial Q}{\partial t}(t, u)=-\frac{\partial}{\partial u} A^{\star} G(u, t)=-\frac{\partial}{\partial u} \frac{\partial}{\partial u} G(u, t)=\frac{\partial}{\partial u} \frac{\partial Q}{\partial t} .
$$

3 а м е ч а н и е 6 . Подчеркнем, что обобщенная функция $Q$ совпадает с бесконечно дифференцируемой функцией вне луча $\{t \geqslant 0, u=0\}$, обращается в нуль на множестве $\{t<0, u<0\}$ и удовлетворяет граничному условию $\lim _{t \rightarrow 0_{+}} Q(t, u)=\delta(u)$. Последнее справедливо, ибо для $t>0$ и гладкой пробной функции $\phi$

$$
\begin{aligned}
\int_{-\infty}^{\infty} Q(t, u) \phi(u) d u & =\int_{0}^{\infty} d u \frac{\partial}{\partial u} \int_{t}^{\infty} G(u, y) d y \phi(u) \\
& =-\int_{0}^{\infty} d u \phi^{\prime}(u) \int_{t}^{\infty} G(u, y) d y
\end{aligned}
$$

что при $t \rightarrow 0$ стремится $\mathrm{\kappa}-\int_{0}^{\infty} \phi^{\prime}(u) d u=\phi(0)$.

Теперь мы обратимся к анализу случайной замены времени $t$ значением процесса $Z(t)$ в марковских процессах.

Теорема 4.2. При условиях теоремь 4.1 пусть $Y(t)$ - феллеровский прочесс в $\mathbf{R}^{d}$, независимьй от $Z(t)$ и с областью определения генератора $L$, содержащей пространство $\left(C_{\infty} \cap C^{2}\right)\left(\mathbf{R}^{d}\right)$. Обозначим переходные вероятности прочесса $Y(t)$ через

$$
T(t, x, d y)=\mathbf{P}\left\{Y_{x}(t) \in d y\right\}=\mathbf{P}_{x}\{Y(t) \in d y\}
$$


Тогда распределение субординированного прочесса $Y(Z(t))$ при $t>0$ определяется формулой

$$
\mathbf{P}_{x}\{Y(Z(t)) \in d y\}=\int_{0}^{\infty} T(u, x, d y) Q(t, u) d u,
$$

средние $f(t, x)=\mathbf{E} f\left(Y_{x}(Z(t))\right)$ функиии $f \in\left(C_{\infty} \cap C^{2}\right)\left(\mathbf{R}^{d}\right)$ удовлетворяют обобщенному дробно-дифференииальному эволюиионному уравнению

$$
A_{t}^{\star} f(t, x)=-L_{x} f(t, x)+f(x) A^{\star} \theta(t)
$$

(где индекс указывает переменную, на которую действует оператор), а их производные по времени $h=\partial f / \partial t$ удовлетворяют при $t>0$ уравнению

$$
A_{t}^{\star} h=-L_{x} h+f(x) \frac{d}{d t} A^{\star} \theta(t) .
$$

Кроме того, если $Y(t)$ обладает гладкой плотностью переходных вероятностей, так что $T(t, x, d y)=T(t, x, y) d y$, и выполняются прямое и обратное уравнения Колмогорова

$$
\frac{\partial T}{\partial t}(t, x, y)=L_{x} T(t, x, y)=L_{y}^{\star} T(t, x, y),
$$

то распределения прочесса $Y(Z(t))$ имеют гладкую плотность

$$
g(t, x, y)=\int_{0}^{\infty} T(u, x, y) Q(t, u) d u
$$

удовлетворяющую прямому обобщенному дробно-дифференииальному уравнению

$$
A_{t}^{\star} g=-L_{y}^{\star} g+\delta(x-y) A^{\star} \theta(t)
$$

и обратному обобщенному дробно-дифференциальному уравнению.

$$
A_{t}^{\star} g=-L_{x} g+\delta(x-y) A^{\star} \theta(t)
$$

(аде g продолжена нулем в область $\{t<0\})$, , а производная по времени $h=\partial g / \partial t$ удовлетворяет уравнению

$$
A_{t}^{\star} h=-L_{y}^{\star} h+\delta(x-y) \frac{d}{d t} A^{\star} \theta(t) .
$$

3 а м е ч а н и е 7 . В случае $\beta$-устойчивого субординатора $X(u)$ с генератором (27)-(28) правые части приведенных уравнений становятся настоящими дробными производными. В частности, если $Y(t)-$ симметричный $\alpha$-устойчивый процесс, то уравнение (35) принимает вид

$$
\frac{\partial^{\beta}}{\partial t^{\beta}} g(t, y-x)=\frac{\partial^{\alpha}}{\partial|y|^{\alpha}} g(t, y-x)+\delta(y-x) \frac{t^{-\beta}}{\Gamma(1-\beta)},
$$


полученный в [21] и [23]. Соответствующий частный случай уравнения (34) имеется в [15], а также и в [21], где он назван формулой разделения переменных. Из нашего общего подхода сразу видно, что такое разделение переменных связано с независимостью процесса $Y(t)$ и субординатора $X(u)$ (см. далее теорему 4.3 , посвященную более общей ситуации).

Д о к а з а т е л ь с т в о. Для непрерывной ограниченной функции $f$ при $t>0$ имеем

$$
\text { E } \begin{aligned}
f\left(Y_{x}(Z(t))\right) & =\int_{0}^{\infty} \mathbf{E}\left(f\left(Y_{x}(Z(t))\right) \mid Z(t)=u\right) Q(t, u) d u \\
& =\int_{0}^{\infty} \mathbf{E} f\left(Y_{x}(u)\right) Q(t, u) d u
\end{aligned}
$$

в силу независимости $Z$ и $Y$. Это влечет (30) и (34).

Из теоремы 4.1 вытекает, что при $t>0$

$$
\begin{aligned}
A_{t}^{\star} g & =\lim _{\varepsilon \rightarrow 0} \int_{\varepsilon}^{\infty} T(u, x, y) A_{t}^{\star} Q(t, u) d u=\lim _{\varepsilon \rightarrow 0} \int_{\varepsilon}^{\infty} T(u, x, y) \frac{\partial}{\partial u} Q(t, u) d u \\
& =-\int_{0}^{\infty} \frac{\partial}{\partial u} T(u, x, y) Q(t, u) d u+\delta(x-y) A^{\star} \theta(t),
\end{aligned}
$$

где, согласно (33), первый член равен $-L_{y}^{\star} g=L_{x} g$, из чего следуют (35) и (36). Разумеется, при $t<0$ обе части уравнений (35) и (36) обращаются в нуль. Остальные уравнения получаются аналогично. Теорема 4.2 доказана.

Теперь мы обобщим теорию на случай феллеровских субординаторов $X(u)$, задаваемых генераторами типа

$$
A f(x)=\int_{0}^{\infty}(f(x+y)-f(x)) \nu(x, d y)+a(x) \frac{\partial f}{\partial x}
$$

с зависящими от позиции мерой Леви и сносом. Разумеется, нужно какое-то условие регулярности, чтобы, как и в случае субординаторов Леви, иметь гладкую переходную вероятность.

Предложение 4.2. Пусть выполнены следующие условия:

(i) $\nu$ имеет плотность $\nu(x, y)$ по отношению $\kappa$ мере Лебега $и$

$$
C_{1} \min \left(y^{-1-\beta_{1}}, y^{-1-\beta_{2}}\right) \leqslant \nu(x, y) \leqslant C_{2} \max \left(y^{-1-\beta_{1}}, y^{-1-\beta_{2}}\right)
$$

с некоторыми постоянными $C_{1}, C_{2}>0$ и $0<\beta_{1}<\beta_{2}<1$;

(ii) $\nu$ трижды непрерывно дифферениируема по х с производными, удовлетворяющими правой оиенке (40);

(iii) а(x) неотричательна и имеет ограниченные производные до третьего порядка.

Тогда генератор (39) задает возрастающий Феллеровский прочесс, имеющий при и >0 плотность переходной вероятности $G(u, y)=$ 
$\mathbf{P}\{X(u) \in d y\}$ (мы предполагаем, ито $X(u)$ стартует в нуле), которая дважды непрерывно дифференцируема по и.

3 а м е ч а н и е 8. Условие (40) выполняется для так называемых процессов устойчивого типа с зависящим от положения индексом устойчивости.

Д оказатель ст о. Существование требуемого феллеровского процесса доказано при более общих предположениях в [1]. Доказательство существования гладкой плотности приведено в [8] при слегка отличных предположениях, но, как легко видеть, приведенные там рассуждения непосредственно переносятся на данную ситуацию.

Ясно, что процесс моментов достижения, определенный формулой $(18)$ с $X(u)$ из предложения 4.1, непрерывен и имеет дифференцируемую плотность $Q(t, u)$ при $t>0$, заданную (22). Однако (23) более не выполняется, ибо оператор $A$ и интегрирование теперь уже не коммутируют. В то же время уравнение (26) остается справедливым (как легко видеть из его доказательства). Отсюда непосредственно вытекает следующее частичное обобщение теоремы 4.2 .

Предложение 4.3. Пусть $Y(t)$ - такой же Феллеровский процесс в $\mathbf{R}^{d}$, как в теореме 4.3, но независимый процесс моментов достижения $Z(t)$ построен по прочессу $X(u)$ при условиях предложения 4.2. Тогда распределения субординированного прочесса $Y(Z(t))$ nрu $t>0$ задаются формулой (30), а производная по времени $h=\partial f / \partial t$ средних $f(t, x)=\mathbf{E} f\left(Y_{x}(Z(t))\right)$ непрерывных ограниченных функиий $f$ удовлетворяет (37).

Наконец, мы обратимся к обобщению на случай моментов достижения, зависящих от субординируемого процесса.

Теорема 4.3. Пусть $(Y, V)(t)$ - случайный прочесс в $\mathbf{R}^{d} \times \mathbf{R}_{+}$ такой, что: (a) компоненты $Y(t), V(s)$ в разные моменты времени имеют совместную плотность вероятностей

$$
\phi\left(s, u ; y_{0}, v_{0} ; y, v\right)=\mathbf{P}_{\left(y_{0}, v_{0}\right)}\{Y(s) \in d y, V(u) \in d v\}
$$

непрерывно диФФерениируемую по и при $u, s>0$, (b) компонента $V(t)$ возрастает и почти наверно не постоянна на любом конечном интервале. (Этим условиям удовлетворяет, например, прочесс из теоремь 3.1.) Тогда:

(i) прочесс моментов достижения $Z(t)=Z_{V}(t)$ (определенный формулой (18) с $V$ вместо $X$ ) почти наверное непрерьвен;

(ii) существует непрерывная совместная плотность вероятности пары $Y(s), Z(t)$, задаваемая формулой

$$
g_{Y(s), Z(t)}\left(y_{0}, 0 ; y, u\right)=\frac{\partial}{\partial u} \int_{t}^{\infty} \phi(s, u ; y, v) d v
$$


(iii) распределение композичии $Y(Z(t))$ имеет плотность вероятности

$$
\begin{aligned}
\Phi_{Y(Z(t))}(y) & =\int_{0}^{\infty} g_{Y(s), Z(t)}\left(y_{0}, 0 ; y, s\right) d s \\
& =\left.\int_{0}^{\infty}\left(\frac{\partial}{\partial u} \int_{t}^{\infty} \phi\left(s, u ; y_{0}, 0 ; y, v\right) d v\right)\right|_{u=s} d s ;
\end{aligned}
$$

(iv) в частности, если $(Y, V)(t)$ - феллеровский прочесс с переходной плотностью вероятности $G_{Y V}\left(u, y_{0}, v_{0}, y, v\right)$ генератором вида $(L+A) f(y, v)$, где $L$ действует на переменную у и не зависит от $v$ (интерпретачия: размер скачка не зависит от времени ожидания), а $A$ действует на $v$ (но может зависеть от $y$ ), то при $s \geqslant u$

$$
\phi\left(s, u ; y_{0}, v_{0} ; y, v\right)=\int G_{Y}(s-u, z, y) G_{Y V}\left(u, y_{0}, v_{0} ; z, v\right) d z
$$

где $G_{Y}$ означает переходную плотность компоненты $Y, u$

$$
\frac{\partial}{\partial t} \Phi_{Y(Z(t))}(y)=\int_{0}^{\infty} A^{\star} G_{Y V}\left(u, y_{0}, 0 ; y, t\right) d u,
$$

т.е. производная по времени от плотности субординированного прочесса равна обобщенной дробной производной «временной компоненты $V \gg$ проинтегрированной совместной плотности процесса $(Y, V)$; производная $h=\frac{\partial}{\partial t} \Phi$ удовлетворяет вместо (37) более сложному уравнению

$$
\left(A^{\star}+L^{\star}\right) h=\delta\left(y-y_{0}\right) A^{\star} \delta(v)+\left[L^{\star}, A^{\star}\right] \int_{0}^{\infty} G_{Y V}\left(u, y_{0}, 0 ; y, v\right) d u .
$$

Д о казател в с т в о. Утверждения (i) и (ii) получаются непосредственным обобщением следствия 1 из предложения 4.1. Утверждение (ii) следует из определения условных и совместных вероятностей. Для доказательства (iv) заметим, что при $s \geqslant u$

$$
\begin{gathered}
\mathbf{E} f\left(Y_{y_{0}}(s), V_{\left(y_{0}, v_{0}\right)}(u)\right)=\mathbf{E} \int G_{Y}\left(s-u, Y_{y_{0}}(u) ; y\right) f\left(y, V_{\left(y_{0}, v_{0}\right)}\right) d y \\
=\iint G_{Y}(s-u, z ; y) f(y, v) G_{Y V}\left(u, y_{0}, v_{0} ; z, v\right) d y d z d v
\end{gathered}
$$

что влечет за собой (44). Следовательно,

$$
\begin{gathered}
\frac{\partial}{\partial t} \Phi_{Y(Z(t))}(y)=-\left.\int_{0}^{\infty} \frac{\partial}{\partial u} \int G_{Y}(s-u, z, y) G_{Y V}\left(u, y_{0}, 0 ; z, t\right) d z\right|_{u=s} d s \\
=\int_{0}^{\infty} \int-\left.\left(L_{z} G_{Y}(s-u, z, y)\right) G_{Y V}\left(u, y_{0}, 0 ; z, t\right) d z\right|_{u=s} d s \\
\quad+\left.\int_{0}^{\infty} \int G_{Y}(s-u, z, y)\left(A^{\star}+L^{\star}\right) G_{Y V}\left(u, y_{0}, 0 ; z, t\right) d z\right|_{u=s} d s
\end{gathered}
$$


откуда вытекает (45), ибо $L$ сокращается в силу сделанных предположений о генераторе. Наконец, (45) непосредственно влечет (46).

5. Предельные теоремы для пространственно неоднородных CTRW. Теперь все готово для нашего главного результата.

Теорема 5.1. $B$ предположениях теорем 3.1 u 3.2 пусть $Z^{\tau}(t)$, $Z(t)$ - прочессы моментов достижения для $V^{\tau}(t / \tau)$ и $V(t)$ (определенные соответствуюшими формулами (18)). Тогда субординированные прочессы $Y^{\tau}\left(Z^{\tau}(t) / \tau\right)$ сходятся $\kappa$ субординированному прочессу $Y(Z(t))$ в смысле маргинальных распределений, т.е.

$$
\mathbf{E}_{x, 0} f\left(Y^{\tau}\left(\frac{Z^{\tau}(t)}{\tau}\right)\right) \rightarrow \mathbf{E}_{x, 0} f(Y(Z(t))), \quad \tau \rightarrow 0
$$

для произвольных $x \in \mathbf{R}^{d}, f \in C_{\infty}\left(\mathbf{R}^{d} \times \mathbf{R}_{+}\right)$, равномерно по $t$ из любого компактного интервала.

3 а м е ч а н и е 9. 1. Распределение предельного процесса описано в теореме 4.3 .

2. Мы доказываем сходимость только в наиболее слабой форме. Не кажется трудным усилить результат до сходимости распределений траекторий из пространства Скорохода, используя стандартные средства (компактность и т.д.) или теорию непрерывных композиций из [24].

3. Подобный результат может быть доказан для непрерывных по времени аппроксимаций из теоремы 3.2 .

Д ок а з а т л в с т в о. Так как время по существу дискретно в $V^{\tau}(t / \tau)$, можно заключить, что $Z^{\tau}(t)=\max \{u: X(u) \leqslant t\}$ и что события $\left\{Z^{\tau}(t) \leqslant u\right\}$ и $\left\{V^{\tau}(u / \tau) \geqslant t\right\}$ совпадают, откуда вытекает, что сходимость конечномерных распределений $\left(Y^{\tau}(s / \tau), V^{\tau}(u / \tau)\right)$ к конечномерным распределениям $(Y(s), V(u))$ (доказанная в теореме 3.3) эквивалентна соответствующей сходимости распределений $\left(Y^{\tau}(s / \tau), Z^{\tau}(t)\right)$ к распределению процесса $(Y(s), Z(t))$.

Далее, поскольку $V(0)=0$, функция $V(t)$ непрерывна, $V(u) \rightarrow \infty$ при $u \rightarrow \infty$, а предельное распределение абсолютно непрерывно, то чтобы доказать (47), достаточно показать, что для достаточно больших $K>0$ и всякого компакта $A$, имеющего границу нулевой меры Лебега, выполнено соотношение

$$
\mathbf{P}_{x, 0}\left\{Y^{\tau}\left(\frac{Z_{K}^{\tau}(t)}{\tau}\right) \in A\right\} \longrightarrow \mathbf{P}_{x, 0}\left\{Y\left(Z_{K}(t)\right) \in A\right\}, \quad \tau \rightarrow 0
$$

где $Z_{K}^{\tau}(t)=Z^{\tau}(t)$, если $K^{-1} \leqslant Z^{\tau}(t) \leqslant K$, и $Z_{K}^{\tau}(t)=0$ в противном случае, и так же определено $Z_{K}(t)$. 
Имеем:

$$
\begin{aligned}
& \mathbf{P}\left\{Y^{\tau}\left(\frac{Z_{K}^{\tau}(t)}{\tau}\right) \in A\right\} \\
& =\sum_{k=1 /(K \tau)}^{K / \tau} \mathbf{P}\left\{V^{\tau}(k) \in A \& Z^{\tau}(t) \in(k \tau,(k+1) \tau)\right\} \\
& \mathbf{P}\left\{Y\left(Z_{K}(t)\right) \in A\right\}=\sum_{k=1 /(K \tau)}^{K / \tau} \int_{A} d y \int_{k \tau}^{(k+1) \tau} g_{Y(s), Z(t)}(y, s) d s
\end{aligned}
$$

Правую часть (50) можно переписать в виде

$$
\begin{aligned}
& \sum_{k=1 /(K \tau)}^{K / \tau} \int_{A} d y \int_{k \tau}^{(k+1) \tau} g_{Y(\tau k), Z(t)}(y, s) d s \\
& \quad+\sum_{k=1 /(K \tau)}^{K / \tau} \int_{A} d y \int_{k \tau}^{(k+1) \tau}\left(g_{Y(s), Z(t)}-g_{Y(\tau k), Z(t)}\right)(y, s) d s
\end{aligned}
$$

Вторая сумма стремится к 0 при $\tau \rightarrow 0$ в силу непрерывности функции (42), а разница между первой суммой и (49) стремится к нулю, поскольку распределения $\left(Y^{\tau}(s / \tau), Z^{\tau}(t)\right)$ сходятся к распределениям $(Y(s), Z(t))$. Отсюда вытекает (48), завершая дохазательство теоремы.

В том случае, когда $S$ не зависит от $u$ и $w$ не зависит от $x$ в (10), предельный процесс $(Y, V)(t)$ имеет независимые компоненты, так что средние субординированного предельного процесса удовлетворяют обобщенному дробн-дифференциальному уравнению из предложения 4.3 , а если к тому же $\boldsymbol{w}$ постолнно, они удовлетворяют дробно-дифференциальному уравнению из теоремы 4.2. В частности, если $p(x, u, d y d v)$ не зависит от $(x, u)$ и разлагается в произведение $p(d y) q(d v)$, причем предельный процесс $V(t)$ устойчивый, из теоремы 5.1 получаются основные результаты работы [15] (в слегка более слабой форме, ибо здесь мы работаем с симметричными устойчивыми законами, а не с операторно устойчивыми, как в [15]), так же как и, разумеется, соответствующие результаты из [7], [11] (надо взять $t=1$ в (47)) об асимптотическом поведении нормализованных субординированных сумм (CTRW) (1).

Я благодарен В.Ю. Королеву, В. Е. Бенингу и В. В. Учайкину, вдохновившим меня красотой CTRW, Дж. Хаттон и Дж. Лэйну, организовавшим мою лекцию о CTRW на Грегиногском статистическом семинаре весной 2007 года, М. М. Меершэрту, привлекшему мое внимание к важной работе [2], а также Х. А. Лопес-Мимбела за гостеприимство и полезные обсуждения в СIMАТ (Мексика, Гуанахуато) летом 2007 года. 


\section{СПИСОК ЛИТЕРАТУРЫ}

1. Bass R.F. Uniqueness in law for pure jump Markov processes. - Probab. Theory Related Fields, 1988, v. 79, № 2, p. 271-287.

2. Becker-Kern P., Meerschaert M. M., Scheffler H.-P. Limit theorems for coupled continuous time random walks. - Ann. Probab., 2004, v. 32, № 1B, p. 730-756.

3. Бенине В.Е., Королев В.Ю., Сухорукова Т.А., Гусаров Г.Г., Саенко В.В., Учайкин $B . B$. Дробно устойчивые распределения. - Стохастические модели структурной плазменной турбулентности. М.: МГУ, 2003, с. 291-360.

4. Bening V.E., Korolev V. Yu., Kolokoltsov V.N., Saenko V.V., Uchaikin V. V., Zolotarev V.M. Estimation of parameters of fractional stable distributions. - J. Math. Sci. (N.Y.), 2004, v. 123, № 1, p. 3722-3732.

5. Bening V.E., Korolev V. Yu., Kolokoltsov V.N. Limit theorems for continuous-time random walks in the double array limit scheme. - J. Math. Sci. (N.Y.), 2006, v. 138, № 1 , p. 5348-5365.

6. Kallenberg O. Foundations of Modern Probability. New York: Springer-Verlag, 2002.

7. Kotulski M. Asymptotic distribution of continuous-time random walks: a probabilistic approach. - J. Statist. Phys., 1995, v. 81, № 3/4, p. 777-792.

8. Kolokoltsov V.N. Symmetric stable laws and stable-like jump-diffusions. - Proc. London Math. Soc., 2000, v 80, № 3, p. 725-768.

9. Kolokoltsov V.N. Semiclassical analysis for diffusions and stochastic processes. Lecture Notes in Math., 2000, v. 1724, p. 1-347.

10. Kolokoltsov V.N. Nonlinear Markov semigroups and interacting Lévy type processes. - J. Statist. Phys., 2007, v. 126, № 3, p. 585-642.

11. Kolokoltsov V. N., Korolev V. Yu., Uchaikin V.V. Fractional stable distributions. J. Math. Sci. (N.Y.), 2001, v. 105, № 6, p. 2569-2576.

12. Королев В.Ю., Бенинг В.Е., Шоргин С.Я. Математические основы теории риска. М.: Физматлит, 2007, 542 с.

13. Korolev V. Yu. et al. Some methods of the analysis of time characteristics of catastrophes in non-homogeneous flows of extremal events. - Системы и средства информатики. Математические модели в информационных технологиях. М.: PAH, 2006, c. 5-23.

14. Маслов В. П. Теория возмущений и асимптотические методы. М.: МГУ, 1965.

15. Meerschaert M. M., Scheffler H.-P. Limit theorems for continuous-time random walks with infinite mean waiting times. - J. Appl. Probab., 2004, v. 41, № 3, c. 623-638.

16. Meerschaert M. M., Scheffler H.-P. Limit Distributions for Sums of Independent Random Vectors. New York: Wiley, 2001, 484 p.

17. Metzler R., Klafter $J$. The random walk's guide to anomalous diffusion: a fractional dynamic approach. - Phys. Rep., 2000, v. 339, № 1, p. 1-77.

18. Miller K.S., Ross B. An Introduction to the Fractional Calculus and Fractional Differential Equations. New York: Wiley, 1993, 366 p.

19. Montroll E. W., Weiss G. H. Random walks on lattices, II. - J. Math. Phys., 1965, v. 6, p. $167-181$.

20. Saichev A.I., Woyczyński W.A. Distributions in the Physical and Engineering Sciences, v. 1. Boston: Birkhäuser, 1997, 336 p.

21. Saichev A.I., Zaslavsky G.M. Fractional kinetic equations: solutions and applications. - Chaos, 1977, v. 7, № 4, p. 753-764.

22. Самарский А. А. Теория разностных схем. М.: Наука, 1989, 432 с.

23. Uchaikin V. V. Montroll-Weiss problem, fractional equations, and stable distributions. - Internat. J. Theoret. Phys., 2000, v. 39, № 8, p. 2087-2105.

24. Whitt W. Stochastic-Process Limits. New York: Springer, 2002, $602 \mathrm{p}$.

25. Zaslavsky G. M. Fractional kinetic equation for Hamiltonian chaos. - Phys. D, 1994, v. 76 , № 1-3, p. 110-122. 